Portland State University

PDXScholar

6-16-2021

\title{
A Hidden Crisis During COVID-19: How Have Intimate Partner Violence Screening Guidelines for Pregnant Patients Changed Since the Start of the Global COVID-19 Pandemic?
}

Zayba Afshar

Portland State University

Follow this and additional works at: https://pdxscholar.library.pdx.edu/honorstheses

Part of the Maternal and Child Health Commons, and the Women's Health Commons Let us know how access to this document benefits you.

\section{Recommended Citation}

Afshar, Zayba, "A Hidden Crisis During COVID-19: How Have Intimate Partner Violence Screening Guidelines for Pregnant Patients Changed Since the Start of the Global COVID-19 Pandemic?" (2021). University Honors Theses. Paper 1116.

https://doi.org/10.15760/honors.1143

This Thesis is brought to you for free and open access. It has been accepted for inclusion in University Honors Theses by an authorized administrator of PDXScholar. Please contact us if we can make this document more accessible: pdxscholar@pdx.edu. 
A Hidden Crisis During COVID-19: How have intimate partner violence screening guidelines for pregnant patients changed since the start of the global COVID-19 pandemic?

by

Zayba Afshar

An undergraduate honors thesis submitted in partial fulfillment of the

requirements for the degree of

Bachelor of Science

in

University Honors

and

Public Health Studies: Pre-clinical Health Science

Thesis Advisor

Dr. Katherine Bradley

Portland State University 


\begin{abstract}
Introduction: Due to the risks of pregnancy outcomes in relation to IPV and the increased rates of IPV as a result of COVID-19 precautions, health care screening for IPV in the prenatal process is even more imperative for preventing negative healthcare outcomes for both the pregnant patient and children.
\end{abstract}

Background: Intimate partner violence (IPV) is a significant public health issue that puts both baby and pregnant patients at risk for severe negative healthcare outcomes. Healthcare screening is a tool to find a condition or to learn more about a patient before symptoms become severe.

Methods: Screening for IPV and the effects of COVID-19 on rates of IPV were reviewed individually. A semi-structured interview was developed to learn more about how obstetricians approach screening of pregnant patients. Twenty-three physicians were emailed for informational interviews.

Results: Three (18.8\%) physicians agreed to be interviewed. Three main themes were identified during the interviews. There was no formal IPV training, the confidentiality necessary to screen a patient for IPV has been compromised since many appointments with pregnant patients have moved from in-person to virtual, and third, the lack of childcare in clinics is a limiting factor for the possibility for pregnant patients to attend regular appointments because of the Covid-19 social distancing restrictions and guidelines.

Conclusion: The biggest barriers found during this research were not training procedures themselves but the uncontrollable variables that have been further complicated by the pandemic. Future research needs to go into the screening practices and capabilities in the virtual arena now that telehealthcare will likely be available beyond the Covid-19 pandemic.

\title{
Introduction
}

The World Health Organization labeled COVID-19 a global pandemic on March 11th, 2020. Since then, there have been countless stay-at-home orders across the United States of America, which has, in turn, played a large part in a surge of Intimate partner violence (IPV) cases due to increased isolation. (Kofman, 2020). There is substantial research connecting the presence of IPV during pregnancy with adverse pregnancy outcomes like preterm birth (PTB) and low birth weight (LBW) (O’Reilly, 2010). Both instances of IPV and COVID-19 are disproportionately prevalent amongst BIPOC (Black, Indigenous, and People of Color) communities (Stockman, 2015 and Valenzuela, 2020). Due to the risks of pregnancy outcomes in relation to IPV and the increased rates 
of IPV as a result of COVID-19 precautions, health care screening for IPV in the prenatal process is even more imperative for preventing negative healthcare outcomes for both the pregnant patient and children. Protocols for screening procedures with the intent to center the safety of potential patients experiencing IPV set long before the start of the global pandemic are now complicated with barriers introduced by COVID-19 thus placing pregnant patients, and especially those identifying as BIPOC, in higher states of risk for negative health outcomes. The purpose of this study is to learn if physicians have had specialized training regarding IPV screening, what screening procedures physicians use during appointments with pregnant patients, how COVID-19 has altered screening practices, and to explore the implications for BIPOC patients.

\section{Background}

Intimate partner violence (IPV) is a significant public health issue that puts both baby and pregnant patients at risk for severe negative healthcare outcomes. The Center for Disease Control (2020) defines IPV as "physical violence, sexual violence, stalking, or psychological harm by a current or former partner or spouse.” Exposure to IPV has been shown to increase the risk of spontaneous abortion, fetal loss, PTB, LBW, and neonatal death (Alhusen, 2015). Approximately 42.4 million (35.6\%) women in the United States have experienced IPV within their lifetime. Intimate partner violence has had a 20\% increase globally during the COVID-19 pandemic (Miller, n.d.). The National Intimate Partner and Sexual Violence Survey indicates the prevalence of IPV amongst non-Hispanic Black and Native American/Alaskan Native women is significantly higher ( $43.7 \%$ and $46 \%$ respectively) compared to non-Hispanic white women (34.6\%) (Stockman, 2015). 
Healthcare screening is a tool to find a condition or to learn more about a patient before symptoms become severe. Regular health care screening is a part of yearly check-ups, for example. Some familiar questions that a primary care provider has most likely asked patients could be: Do you have a family history for diabetes? Do you use tobacco? How often do you drink alcohol? How many sexual partners do you have? Etc. Most healthcare providers should ask similar questions specifically geared toward assessing the possibility of IPV in a pregnant patient.

\section{Literature Review}

In 1992 the American Medical Association (AMA) recommended that all women who access primary care (and some secondary care facilities) get screened for IPV (American Medical Association Diagnostic and Treatment Guidelines on Domestic Violence, 1992). However, despite recommendations, the approach to screening for IPV during pregnancy is not universal. The CDC in 2004 concluded that "the extent to which US clinicians incorporate screening for IPV into their practices is relatively unknown" (Bailey, 2010). More recently in 2015, despite the recommendations of the United States Preventive Services Task Force (USPSTF), studies have shown that only 1.5\%-12\% of pregnant patients get screened for IPV despite the fact that $96 \%$ receive prenatal care (Intimate Partner Violence Screening, 2015). Screening is a tool to identify IPV in a pregnant patient which allows providers a unique ability to detect IPV early due to elongated relationships with the patient throughout their pregnancy. If IPV is detected early during screening it may even help prevent adverse effects on maternal and pregnancy outcomes. In a systematic literature review, Bailey (2010) found at that time 
numerous organizations and healthcare providers had recommended a universal screening procedure, but there did not appear to be a universal practice in place.

The 2017 Women's Health Survey conducted by the Kaiser Family Foundation (KFF) shows that in 2017 only $27 \%$ of women reported they were screened for IPV with a medical provider and women who were low-income, on Medicaid, and/or identified as Black or Latina were the most likely to be screened (Gomez et al., 2018). Researchers went on to specify that Latina women and women covered by Medicaid were significantly more likely to seek care from community health centers or clinics and argued that clinics are essential to the care of women of color. Community health centers that frequently serve marginalized populations would know the barriers their patients are predisposed to thus offering a possible reason why women on Medicaid and/or Black and Latina women were more likely to be screened.

The U.S. Preventive Services Task Force (USPSTF) reviewed a number of screening tools and came up with a list of six they recommended for their sensitivity and specificity: HITS (Hurt, Insult, Threaten, Scream), OVAT (Ongoing Violence Assessment Tool), STaT (Slapped, Things, and Threaten), HARK (Humiliation, Afraid, Rape, Kick), CTQ-SF (Modified Childhood Trauma Questionnaire- Short Form), WAST (Women Abuse Screen Tool) (Agency for Healthcare Research and Quality [AHRQ], 2015). Four of the six screening tools are discussed in a PDF document from the CDC in a collection of almost one hundred other screening tools designed by variations of doctors, non-profit organizations, universities, clinics, and hospitals (Center for Disease Control [CDC], 2007). The STaT screening tool must be purchased from an outside organization but is referenced. Each one uses a scoring process to determine the level of risk. Providers without training or extensive knowledge may have a difficult time 
understanding the differences, deciding which one to use, and learning how to implement with the safety of a patient in mind. The CDC's endorsement of so many screening tools rather than a set of universally recommended standards runs the risk of confusing providers and causing a lowered rate of screening or, more dangerously, improper screening that could put the patient in further danger.

The Agency for Healthcare Research and Quality (AHRQ) recommends numerous screenings for IPV at "periodic intervals, including obstetric care at the first prenatal visit, at least once per trimester, and at the postpartum checkup" which is in accordance with other official organizations such as the United States Department of Health and Human Services (HHS) and the Institute of Human Medicine (IOM) (AHRQ, 2015). Authors Ramaswamy, Ranji, and Salganicoff (2019) also discuss IPV screening tools in their recent study published by the Kaiser Family Foundation (KFF) in which they agree with the AHRQ's recommendations for screening intervals and further explore barriers to the screening process. They argue that the main barriers providers face with regards to IPV screening is the presence of a partner, lack of time to build a relationship that would encourage patients to disclose, patients' concerns about mandatory reporting laws, and lack of adequate knowledge about the issue of IPV and screening procedures itself (possibly explained by the number of screening tools available to them rather than a clear set of universal guidelines).

The COVID-19 pandemic forced national stay-at-home mandates across the United States which have forced victims of IPV to be isolated with their abusers. Intimate partner violence hotlines expected to see states increase the demand for their services. However, many organizations have seen major declines in calls which is an alarming observation (Evans, 2020). This indicates that people do not feel safe enough 
to call IPV hotlines. Organizations such as the World Health Organization (WHO) have already conducted extensive research and published articles regarding the intersection of COVID-19 with instances of IPV. The WHO examined variables that exacerbated the risks for violence against women and found that increased stress (mental, physical, and financial) accompanied by the stay-at-home orders accounts for the greatest increase in risk (World Health Organization [WHO], 2020). Isolation and social distancing have left many in the United States unable to see friends and family who, for many women experiencing IPV, may be the only source of support and safety they have. Women may also have a difficult time connecting to community centers, hotlines, and healthcare due not only to the constant presence of abusers but also lowered services as organizations attempt to protect their employees. Abusers have traditionally exhibited control by taking away access to necessary items and the WHO stated that "perpetrators may also restrict access to necessary items such as soap and hand sanitizer... exert control by spreading misinformation about the disease and stigmatize partners" (WHO, 202O, p. 1) A representative from the National Domestic Violence Hotline (NDVH) shared anonymous stories about calls since the start of the pandemic that corroborate the WHO's research with a journalist from The Guardian (Fielding, 2020). According to the representative, 951 callers between March 10th and March 24th, 2020 mentioned COVID-19. Fielding wrote of the calls,

One caller to a domestic violence hotline reported that her husband threatened to throw her out into the street if she coughed. Another reported they had been strangled by their partner, but feared going to the hospital because of the threat of coronavirus. An immunocompromised man from Pennsylvania called in after his emotionally abusive girlfriend began hiding cleaning supplies and hand sanitizer from him. 
The WHO also offered recommendations for policymakers, health care providers, humanitarian organizations, community members, and women experiencing violence in the wake of new COVID-19 barriers, but it is on individual systems and people to take on the work (WHO, 2020).

\section{Methods}

Terms and descriptions have been carefully considered in the planning and execution of this thesis. 'Intimate partner violence' is used instead of its commonly used counterpart 'interpersonal violence' as the latter refers to violence between individuals whereas the former touches on an intimate relationship putting potential strain on a pregnancy and the screening of IPV. Domestic violence was not used as this term is specific to relationships in which all parties live in the same household. Patients are referred to as 'pregnant patients' in the context of this research while terminology in the literature review reflects the terminology used in the original source. This decision was made to encompass the wide spectrum of people who can become pregnant and avoid assuming gender. The acronym 'BIPOC' is used as opposed to the common 'POC' it highlights the specific experiences that Black and Indigenous peoples have experienced over the course of history.

This research paper started through a preliminary literature review. The intersection of COVID-19 and IPV was well-researched prior to the beginning of this project, though its conjoined effects on screening were not discussed. Screening for IPV and the effects of COVID-19 on rates of IPV were reviewed individually, the former reviewed first. This included researching types of screening procedures, implementation 
of screening procedures, barriers to screening procedures, and the similarities and differences in cases of IPV before and during the pandemic.

After establishing the purpose of this research through a literature review, a semi-structured interview was developed to learn more about how OB/GYNs approach screening pregnant patients. The project proposal was reviewed by the Portland State University IRB and granted exemption approval. Twenty-three physicians were emailed for informational interviews from a large healthcare organization. This study only sought out practicing physicians with medical degrees. The physicians were all sent a copy of the consent procedure and interview questions. The physicians who were interviewed were sent a Webex link for a virtual meeting. The interviewees gave their verbal consent at the start of the interview. The audio and video from the interviews were recorded via Webex. Additionally, the software recorded a written transcription of the interviews. The responses were analyzed via content analysis to identify key themes. After the key themes were extracted from the data the recorded audio, video, and transcription data were destroyed.

The content analysis consisted of creating a spreadsheet organized based on interviewee and question number. The interview responses were listed in order and segments were pulled out. From there specific phrases were identified to hone in on key themes. Three themes were identified followed by a limited selection of important points that proved necessary to consider as well. 


\section{Results}

Out of the twenty-three physicians contacted, sixteen (69.65\%) responded. Of the sixteen physicians who responded, three (18.8\%) physicians provided recommendations for other potential interviewees. Three (18.8\%) physicians who responded to the recruitment email, agreed to be interviewed. Three main themes were unanimously identified during all of the interviews. The first being that there was no formal training in regards to IPV training. All three physicians indicated that they had training for IPV screening at some point during their training but had no formal training. The second theme was that the confidentiality necessary to screen a patient for IPV has been largely compromised since many appointments with pregnant patients have moved from in-person to virtual due to the social distancing rules put into place. This negatively impacts IPV disclosure. The last theme was that the lack of childcare in clinics is a limiting factor for the possibility for pregnant patients to attend regular appointments because of the Covid-19 social distancing restrictions and guidelines. One of the interviewees went as far as to say that the issue should be 'yelled about from rooftops'. Additionally, a physician indicated a key patient population not included in this study is the patient population seeking to terminate their pregnancy.

All physicians indicated the use of a standardized script in their practices which has increased the frequency of the screening capabilities since the start of the Covid-19 pandemic. In regards to if Covid-19 had increased the instances of IPV in their patients, the responses were mixed. Some spoke to the increased prevalence anecdotally and others stated that there has not been a broad investigation into if there has been an increased prevalence of IPV in their patients so the answer is unknown. 


\section{Discussion}

A significant barrier to providers' screening procedures is a lack of formal training for screening for IPV in pregnant patients for the physicians. The formal training should consist of guidance on how to navigate the conversation, and how to counsel and refer patients who have disclosed that they are experiencing IPV (Schrier, 2017). A module that incorporated these two main themes saw a high rating from $90 \%$ of the 260 second-year medical students who utilized it. The lack of formal training can leave providers ill-equipped and uncomfortable when having a difficult conversation like this which may offer negative impacts on the rate of conducted screenings.

Due to the nature of the Covid-19 pandemic, many appointments have been shifted to the virtual realm making it more likely that patient partners are present or privacy is not guaranteed, something that may happen if a patient must stay home due to a lack of childcare. This then flags the appointment because the provider was unable to screen the patient for IPV due to the risk of placing the patient in a dangerous situation. Lack of privacy also negatively impacts the possibility of disclosure. This is important because the provider is unable to screen the patient and ensure privacy as they might otherwise be able to do in a private room in their clinic. The implication is that patients will not be screened despite a possibility of being IPV positive, ultimately risking serious negative health outcomes in addition to their growing fetus.

Limited access to childcare was listed as a large impact on patients' ability to attend meetings with physicians. The social distancing guidelines have closed the in-house child care services previously available, meaning that patients have to look for 
other childcare arrangements. This could increase travel time and be a limitation to be able to attend a meeting. One of the interviewees indicated that the lack of childcare services is a large barrier for, particularly, BIPOC patients. BIPOC patients might have to work more and work in lower-paying jobs across the board due to systemic racism and lack of generational wealth.

\section{Conclusion}

There is conflicting data on the importance of universal screening procedures. The physicians interviewed had a consensus that screening is easier now due to standardized practices, while sources in the literature found that the lack of universal procedures (despite standardized practices) offers little accountability and limited guidance. The biggest barriers found from the interviews were not the procedures themselves, but uncontrollable variables complicating the completion of the screening. It is the uncontrollable variables that have been further complicated by the pandemic and the introduction of telehealth medicine. These barriers will be difficult to counteract as they are unique to the patients and the systems they work within (i.e. access to childcare, partners who work from home, access to transportation, a clinic's set up, and ability to expand care). However, one interviewee pointed out that nurses took on a bit more in an attempt to complete screening. When a patient was flagged due to an incomplete screening via a telehealth appointment, a nurse would then conduct a follow-up phone call. If confidentiality was achieved, screening was conducted. Gomex et al. found that utilizing the relationship with a nurse to counteract time constraints in an appointment has increased the rate of screening. It seems that the relationship 
between the nurse, the physician, and the patient is an important variable that can be capitalized on for telehealth screening procedures.

A major limitation is that three people were interviewed. Those three people were all OB/GYN physicians from one practice. Further research should conduct more interviews with a wider variety of healthcare professionals and from different practices. Additionally, all the physicians were a part of the same organization and served a pregnant patient population that chose not to terminate their pregnancies Further research should explore that population because their experiences with IPV could be completely different. Future research needs to go into the screening practices and capabilities in the virtual arena now that telehealth care will likely be available beyond the Covid-19 pandemic. 


\section{Bibliography}

Agency for Healthcare Research and Quality. (n.d.). Intimate Partner Violence Screening. https://www.ahrq.gov/ncepcr/tools/healthier-pregnancy/fact-sheets/partner(Intimate Partner Violence Screening , 2015).html

Ahmed, S. (2011). Effects of Domestic Violence on Perinatal and Early-Childhood Mortality: Evidence From North India. Publication of the American Public Health Association.

Alhusen, J. L. (2015). Intimate Partner Violence During Pregnancy: Maternal and Neonatal Outcomes. Journal of Women's Health, 100-106.

American Medical Association Diagnostic and Treatment Guidelines on Domestic Violence. (1992). The American College of Obstetricians and Gynecologists, $39-47$.

Bailey, B. A. (2010). Partner violence during pregnancy: prevalence, effects, screening, and management. International Journal Women's Health, 183-197.

Center for Disease Control. (2007). Intimate Partner Violence and Sexual Violence Victimization Assessment Instruments for Use in Healthcare Settings. https://www.cdc.gov/violenceprevention/pdf/ipv/ipvandsvscreening.pdf

Centers for Disease Control and Prevention. (2020). Intimate Partner Violence. https://www.cdc.gov/violenceprevention/intimatepartnerviolence/index.html

Evans, M. L., Lindauer, M., Farrell, M. E. (2020). A Pandemic within a PandemicIntimate Partner Violence during Covid-19. The New England Journal of Medicine. DOI: 10.1056/NEJMp2024046

Fielding, S. (2020). In quarantine with an abuser: surge in domestic violence reports linked to coronavirus. The Guardian. https://www.theguardian.com/us-news/2020/apr/o3/coronavirus-quarantine-a buse-domestic-violence

Gomez, I., Ranji, U., Rosenzweig, C., Salganicoff, A. (2018). Overview: 2017 Kaiser Women's Health Survey. Kaiser Family Foundation. https://www.kff.org/report-section/executive-summary-2017-kaiser-womens-he alth-survey/

Agency for Healthcare Research and Quality. (2015, April). Intimate Partner Violence Screening. (2015, April). https://www.ahrq.gov/ncepcr/tools/healthier-pregnancy/fact-sheets/partner-vi olence.html 
Kofman, Y. (2020). Home is not always a haven: The domestic violence crisis amid the COVID-19 pandemic. US National Library of Medicine National Institutes of Health, p199-201.

Miller, K. (n.d.). Intimate Partner Violence during the COVID-19 Pandemic. Legacy Community Health:

https://www.legacycommunityhealth.org/newsblog-intimate-partner-violence-d uring-the-covid-19-pandemic/\#: :text=We\%2ohave\%20all\%2oheard\%20the,(IP V)\%20within\%20their\%2olifetime.\&text=That\%2omeans\%2C\%2ofor\%2oevery \%20three,cases\%20of\%20IPV\%20will\%20occur

Ramaswamy, A., Ranji, U., Salganicoff, A. (2019, December 2). Intimate Partner Violence (IPV) Screening and Counseling Services in Clinical Settings. Kaiser Family Foundation.

https://www.kff.org/womens-health-policy/issue-brief/intimate-partner-violenc e-ipv-screening-and-counseling-services-in-clinical-settings/

O'Reilly, R. (2010). Screening and Intervention for Domestic Violence During Pregnancy Care: A Systematic Review. SAGE Journals.

Schrier, M. W., Rougas, S. C., Schrier, E. W., Elisseou, S., \& Warrie, S. (2017). Intimate Partner Violence Screening and Counseling: An Introductory Session for Health Care Professionals. MedEdPORTAL : the journal of teaching and learning resources, 13, 10622. https://doi.org/10.15766/mep_2374-8265.10622

Stockman, J. K. (2015). Intimate Partner Violence and Its Health Impact on Disproportionately Affected Populations, Including Minorities and Impoverished Groups. Journal of Women's Health, 62-79.

Valenzuela, J. (2020). Commentary: Reflections on the COVID-19 Pandemic and Health Disparities in Pediatric Psychology. Journal of Pediatric Psychology, 839-941.

World Health Organization. (2020, March 26). COVID-19 and violence against women: What the health sector/system can do. https://www.who.int/reproductivehealth/publications/emergencies/COVID-19VAW-full-text.pdf 